Heiner Martin*, Guido Schröder, Julia Schubert, Anne Ringel, Jorid Paulsen, Sylvia Pfensig, Andreas Götz, Hans Christof Schober, Niels Grabow

\title{
A two-stage finite element based method for the study of stiffness and failure load of osteoporotic spines
}

\begin{abstract}
A two-step method for investigating the stiffnesses and failure loads of vertebral bodies is presented. It is based on the representation of the spine in a global finite element model of structural elements. A detailed model of two interconnected vertebrae and an intervertebral disc was implemented. Calculations on this model are shown which illustrate the dependencies of structural stiffnesses on vertebral body properties. These structural stiffnesses can then be used to represent the spine in the global model.
\end{abstract}

Keywords: Biomechanics, spine, Finite element modeling, osteoporosis

https://doi.org/10.1515/cdbme-2021-2151

\section{Introduction}

When investigating the causes of spinal damage and failure, it is desirable to examine the entire spine, including the ligamentous apparatus and intervertebral discs. Finite element models of spinal segments are known from the literature [1]. However, these models are still too complex for investigating the mechanics of the entire spine, because a sufficiently fine meshing of the vertebral bodies and the intervertebral discs is required. On the other hand, it is of interest to model the exact mechanism of load transmission within the spine as accurately as possible. Finite element models are suitable for this purpose, which simulate the

\footnotetext{
*Corresponding author: Heiner Martin: Institute for Biomedical engineering, University Medical Centre, Rostock, F.-Barnewitz-Str. 4, Rostock, Germany, e-mail: heiner.martin@uni-rostock.de Guido Schröder: Clinic for Surgery, Department of Orthopedics and Trauma Surgery, Buetzow, Germany Julia Schubert, Anne Ringel, Jorid Paulsen, Andreas Götz, Niels Grabow: Institute for Biomedical engineering Rostock, University Medical Centre, Rostock, Germany Sylvia Pfensig: Institute for Implants and Biomaterials e.V., Rostock, Germany

Hans-Christof Schober: Clinic for Internal Medicine I, Southern Clinical Centre, Rostock, Germany
}

spinal column with structural elements including intervertebral discs and ligaments (Fig. 1).

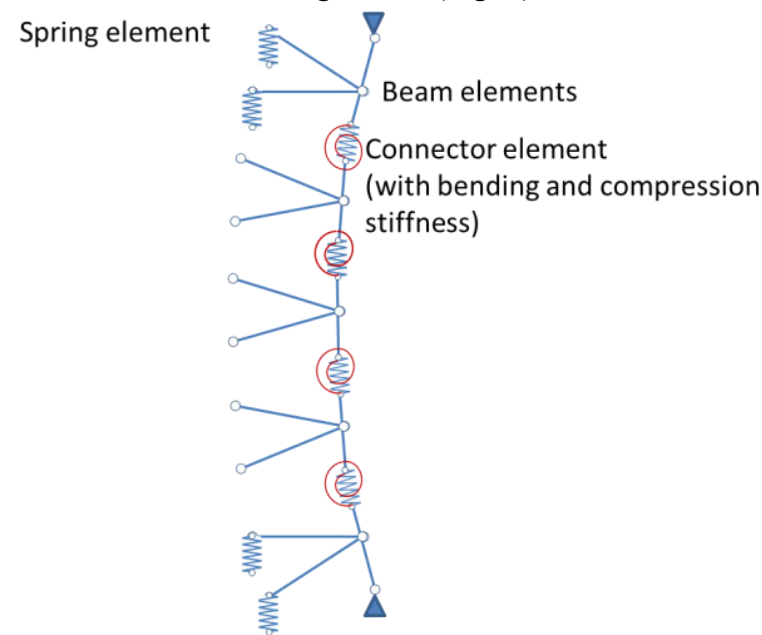

Figure 1: Schematic view of the global model of the spine

\section{Method}

In this paper, a simplified finite element model of the entire spine made of structural elements is proposed. For FEA, Abaqus/Standard 2020 (Dassault systems, VelizyVillacoublay) was used. It contains the vertebral bodies, which are represented by rod elements including the spinous processes, the intervertebral discs and the ligamentous apparatus of the spine, which are represented by rod, connector and spring elements. The spring and torsion spring constants are determined from detailed finite element models of two vertebral bodies with intervertebral discs, which are investigated in compression and bending load cases. These models are generated from parametric vertebral body geometries similar to Lavaste et al [2], which are adapted in the main dimensions to the respective spinal region. The main dimensions for the vertebral body models are taken from $\mathrm{CT}$ datasets obtained by examining cadaveric spine sections in water phantoms. 


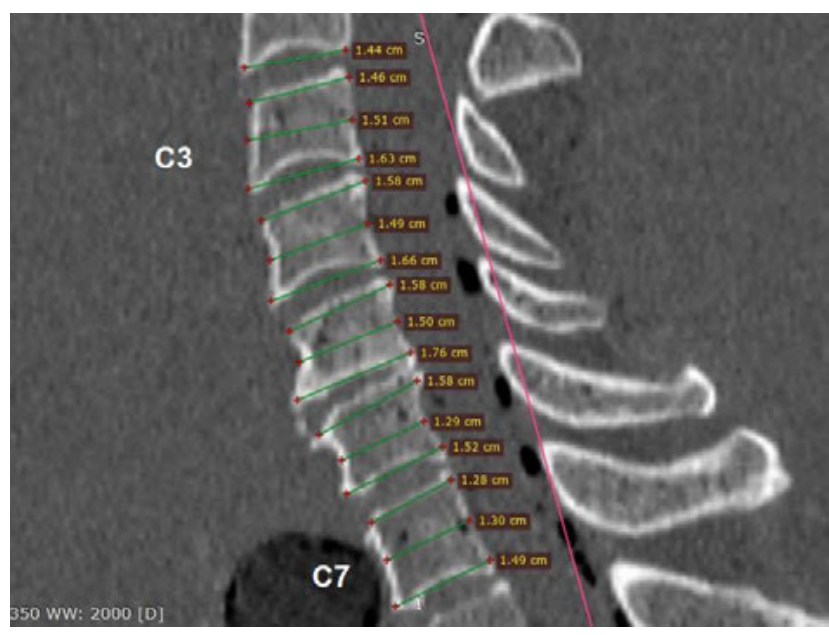

Figure 2: Sagittal section of vertebral WS 21 showing two dimensional determination of main dimensions

Fig. 2 shows an image of such a data set. It can be seen that the datasets allow determination of the main dimensions of vertebral bodies. However, detailed determinations of the thickness of the cortical bone and the cancellous bone structure are not possible with such data sets. Therefore, the cancellous bone was modelled as a continuum and the cortical bone as a uniform shell.

The vertebral body main dimensions were set in the model in the order of magnitude of the values from the evaluation of the radiological data. (See Fig. 3). This model was designed using the CAD software Creo Parametrics 6.0 (PTC Inc, Boston, MA, USA).

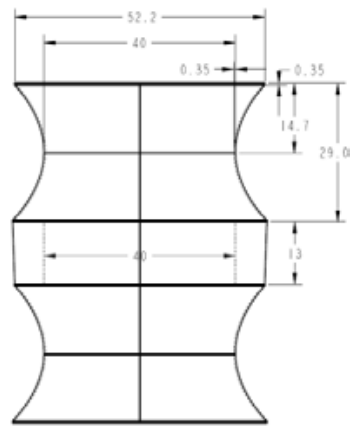

Figure 3: Frontal view showing dimensions of simplified model of vertebrae

The simplified parametric model of two vertebral bodies connected by intervertebral disc (Fig. 4) consists of vertebral bodies characterised by the following essential parameters:

- Young's moduli of the cortical bone $\mathrm{E}_{\mathrm{c}}=12700 \mathrm{MPa}$ [4] and the cancellous bone (structural Young's modulus) $\mathrm{E}_{\mathrm{s}}=100 \mathrm{MPa}$ [5].

- Young's moduli of the intervertebral disc: Nucleus $\mathrm{E}_{\mathrm{n}}=0.15 \mathrm{MPa}$ and Annulus $\mathrm{E}_{\mathrm{a}}=1.5 \mathrm{MPa} ; \mathrm{v}=0.17$ [3].

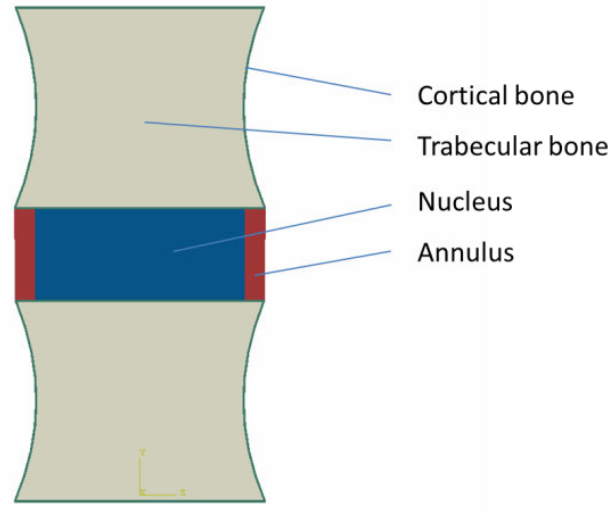

Figure 4: Sagittal section of the detail model, showing the different materials

The spine is subject to a combined load. Of particular interest are the compressive and bending loads on the spine and the stiffnesses against these loads. They were determined by determining the load-displacement curve and by determining the increase in the linear range. The stiffnesses against compression and bending were determined, which in turn are used as input values for the global model of the spine.

\section{Results}

Model calculations for stiffnesses of various vertebral body parameters are presented. The essential dependencies of the stiffnesses on the main dimensions of the vertebral bodies become clear. The models allow statements about the stiffness of the spinal column sections and can be used in the global spinal column model.

The determined stiffnesses are in the order of magnitude of experimentally determined values for vertebral bodies [5] The load-displacement curve of two interconnected vertebral bodies shows an almost linear course, so that the compressive stiffness of the entire vertebral body can be determined from the increase.

The following dependencies were determined:

1. Dependence of the compressive stiffness of the vertebral body on the elastic modulus of the cancellous bone (Fig. 5)

2. Dependence of the compressive stiffness on the elastic modulus of the cortical bone (Fig. 6).

Fig. 5 shows that the compressive stiffness of the entire vertebral body only decreases at very low elastic moduli of the cancellous bone, while the stiffness remains constant over a large range. Stiffness of cortical bone is constant for each analysis. 


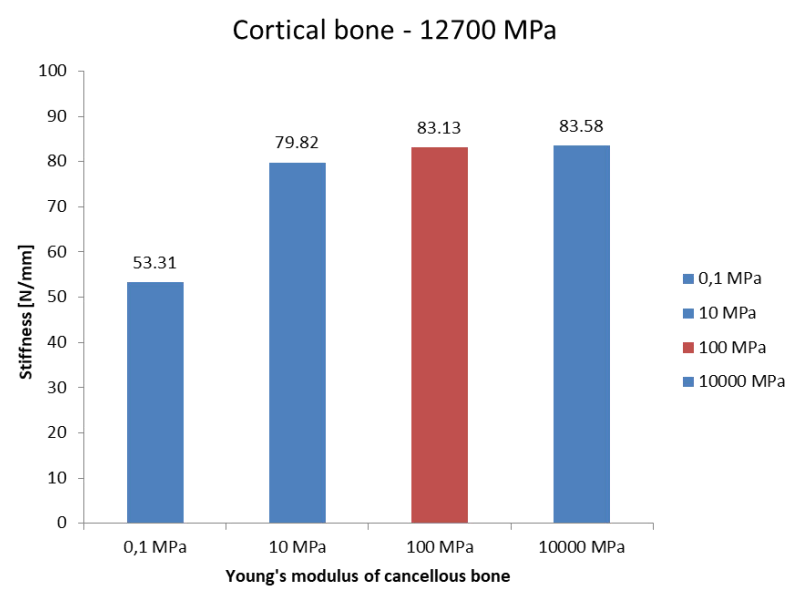

Figure 5: Stiffness against pressure load depending on the cancellous bone Young's modulus (red bar: based on [5])

Fig. 6 shows that the elastic modulus of the cortical bone has little influence on the overall stiffness of the vertebral body when the spongiosa stiffness remains constant. Hence, it can be concluded that the compressive stiffness of the vertebral body is mainly influenced by the stiffness of the cancellous bone and that the cortical bone mainly performs the function of load application and ensuring cohesion.

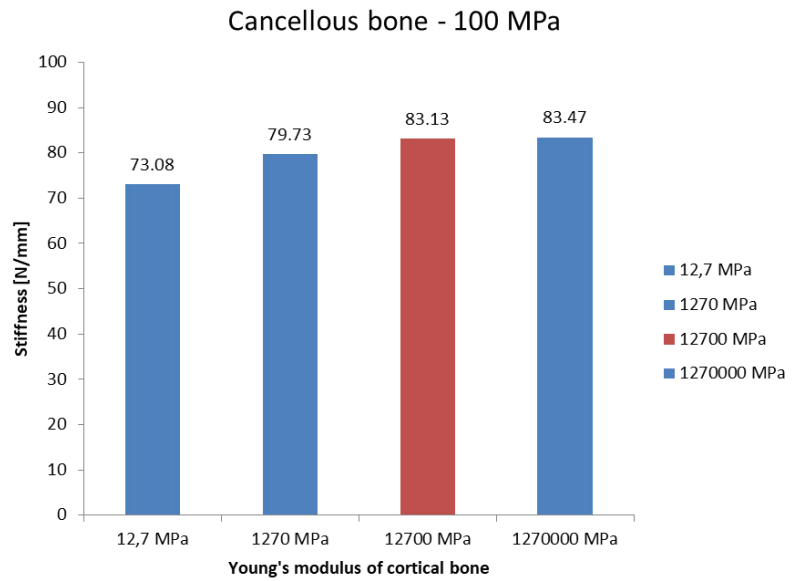

Figure 6: Stiffness against pressure load depending on the Young's modulus of cortical bone (red bar: based on [4])

Thus, the essential determinants for a global spine model can be determined, which will be investigated in a next step.

\section{Discussion}

Y. Schröder [3] found that the water content of the intervertebral discs results in changes in the stiffness of the composite of vertebral bodies. These changes in stiffness are only considered here via the changes in the material properties of the intervertebral disc. It is also undisputed that a complete investigation of the mechanics of the spinal column requires consideration of the ligaments. These influences were also neglected here for the time being, but will be taken into account during the further development of the model.

\section{Conclusion}

The investigations show the feasibility in principle of the proposed methodology. Dependence of the spring constants on structural parameters was determined and presented.

For further development, an investigation on the complete model is necessary as a next step. The focus here is on the identifiability of failure mechanisms that allow statements about the permissible spinal load. A refinement of the detailed model towards more realism (inclusion of the facet joints) is also planned.

\section{Author Statement}

Research funding: The author state no funding involved. Conflict of interest: Authors state no conflict of interest. Informed consent: Informed consent has been obtained from all individuals included in this study. Ethical approval: The research related to human use complies with all the relevant national regulations, institutional policies and was performed in accordance with the tenets of the Helsinki Declaration, and has been approved by the authors' institutional review board or equivalent committee.

\section{References}

[1] Rohlmann A, Zander T, et al.: Applying a follower load delivers realistic results for simulating standing. J Biomech. 2009 Jul 22;42(10):1520-1526

[2] Lavaste F, Skalli W et al.: Three-dimensional Geometrical and Mechanical Modelling of the Lumbar Spine. J Biomech 1992 Oct;25(10):1153-64

[3] Schröder Y: Putting pressure on the spine - an osmoviscoelastic FE model of the intervertebral disc. Diss. 2007, TU Eindhoven, ISBN 978-90-386-1187-7.

[4] Tensile Young's modulus for cortical human Femur: 17.6 GPa, cf. Y.C. Fung: Biomechanics, Mechanical Properties of Living Tissues. Springer 1993, p. 511.

[5] J. Werner: Die nichtinvasive Ermittlung von Steifigkeit und Versagenslast humaner Wirbelkörper. Shaker Verlag Aachen 1996 ISBN 3-8265-1992-2. 